\title{
Erratum: A molecular phylogeny of the Lecanora varia group, including a new species from western North America
}

Sergio Pérez-Ortega • Toby Spribille • Zdeněk Palice • John A. Elix • Christian Printzen

Published online: 1 December 2010

(C) German Mycological Society and Springer 2010

\section{Erratum to: Mycol Progress}

DOI 10.1007/s11557-010-0660-y

The original version of this article unfortunately contained a mistake. The presentation of Table 1 was incorrect. The corrected Table 1 is given below.

The online version of the original article can be found at http://dx.doi. org/10.1007/s11557-010-0660-y.

S. Pérez-Ortega $\cdot$ C. Printzen

Abt. Botanik und Molekulare Evolutionsforschung,

Forschungsinstitut Senckenberg,

60325 Frankfurt am Main, Germany

T. Spribille

Albrecht-von-Haller-Institut für Pflanzenwissenschaften,

Universität Göttingen,

Untere Karspüle 2,

37073 Göttingen, Germany

Z. Palice

Institute of Botany, Czech Academy of Sciences,

25243 Průhonice, Czech Republic

\section{Z. Palice}

Department of Botany, Faculty of Natural Sciences,

Charles University,

Benátská 2,

12801 Praha, Czech Republic
J. A. Elix

Research School of Chemistry, Building 33,

Australian National University,

Canberra ACT 0200, Australia

Present Address:

S. Pérez-Ortega $(\square)$

Centro de Ciencias Medioambientales (CSIC),

c/Serrano 115 dpdo,

E-28006 Madrid, Spain

e-mail: sperezortega@ccma.csic.es

Present Address:

T. Spribille

Institute of Plant Sciences, University of Graz,

Holteigasse 6 ,

8010 Graz, Austria 
Table 1 Species of Lecanora, their origins, collectors, herbarium and accession numbers

\begin{tabular}{|c|c|c|c|}
\hline Species & Country/State/Province & Collector, Herbarium & Accesion number \\
\hline Lecanora achariana & & & AF070019 \\
\hline L. aitema & Germany, Hessen & Ch. Printzen s.n. (FR) & GU480092 \\
\hline Lecanora allophana & & & AF159939 \\
\hline Lecanora anopta 1 & Czech Republic & Z. Palice s.n. (hb. Palice) & GU480114 \\
\hline Lecanora anopta 2 & Canada, British Columbia & T. Spribille 16580 (FR) & GU480115 \\
\hline Lecanora anopta 3 & Canada, Yukon Territory & T. Spribille 25131 (hb. Spribille) & GU480116 \\
\hline L. austrocalifornica & U.S.A., Arizona & T.H Nash 43991 (ASU) & GU480103 \\
\hline Lecanora burgaziae 1 & Spain, Cáceres & S. Pérez-Ortega LB46 (FR) & GU480117 \\
\hline Lecanora burgaziae 2 & Spain, Toledo & S. Pérez-Ortega LB61 (FR) & GU480118 \\
\hline Lecanora campestris & & & AF159930 \\
\hline Lecanora concolor & & & AF070037 \\
\hline Lecanora confusa 1 & U.K., Scotland & B.J. \& A.M. Coppins s.n. (E) & GU480120 \\
\hline Lecanora confusa 2 & U.S.A., Montana & S. Pérez-Ortega 1158 \& T. Spribille (FR) & GU480093 \\
\hline Lecanora conizaeoides & & & AF189717 \\
\hline Lecanora densa 1 & U.S.A., Colorado & S. Pérez-Ortega LD221 (FR) & GU480094 \\
\hline Lecanora densa 2 & U.S.A., New Mexico & S. Pérez-Ortega LD185 (FR) & GU480095 \\
\hline Lecanora densa 3 & U.S.A., Arizona & S. Pérez-Ortega LD171 (FR) & GU480096 \\
\hline Lecanora densa 4 & U.S.A., Montana & S. Pérez-Ortega LD128 \& T. Spribille (FR) & GU480097 \\
\hline Lecanora dispersoareolata & & & AF070016 \\
\hline Lecanora epibryon & & & DQ534469 \\
\hline Lecanora aff. expallens & Denmark, Hovedstaden & S. Pérez-Ortega 1170 \& U. Søchting (FR) & GU480098 \\
\hline Lecanora filamentosa 1 & Germany, Niedersachsen & R. Cezanne \& M. Eichler 6791 (FR, as Lecidea hercynica, topotype) & GU480099 \\
\hline Lecanora filamentosa 2 & Czech Republic, W. Bohemia & Ch. Printzen s.n. (FR) & GU480100 \\
\hline Lecanora flavoleprosa & Poland & P. Czarnota s.n. (GPN) & GU480101 \\
\hline Lecanora cf. fulvastra & Cuba, Pinar del Río & S. Pérez-Ortega 1159 (FR) & GU480119 \\
\hline Lecanora garovaglii & & & AF189718 \\
\hline Lecanora intricata & & & AY398703 \\
\hline Lecanora laxa 1 & U.S.A., Colorado & S. Pérez-Ortega LL34 (FR) & GU480102 \\
\hline Lecanora laxa 2 & U.S.A., Colorado & S. Pérez-Ortega LL35 (FR) & GU480104 \\
\hline Lecanora laxa 3 & U.S.A., Colorado & S. Pérez-Ortega LL36 (FR) & GU480105 \\
\hline Lecanora laxa 4 & U.S.A., Colorado & S. Pérez-Ortega LL2 (FR) & GU480106 \\
\hline Lecanora laxa 5 & U.S.A., Colorado & S. Pérez-Ortega LL4 (FR) & GU480107 \\
\hline Lecanora muralis & & & AF159922 \\
\hline Lecanora novomexicana & & & AF159923 \\
\hline Lecanora opiniconensis & & & AF159928 \\
\hline Lecanora orosthea & & & AY398701 \\
\hline Lecanora perpruinosa & & & AF070025 \\
\hline Lecanora polytropa 1 & & & DQ534470 \\
\hline Lecanora polytropa 2 & & & AF070017 \\
\hline Lecanora pruinosa & & & AF070018 \\
\hline Lecanora saligna 1 & U.S.A., New Mexico & S. Pérez-Ortega 1161 (FR) & GU480108 \\
\hline Lecanora saligna 2 & & & AF189716 \\
\hline Lecanora schizochromatica 1 & Canada, British Columbia & T. Spribille 17016 (hb. Spribille) & GU480109 \\
\hline Lecanora schizochromatica 2 & Canada, British Columbia & T. Spribille 16850-A (hb. Spribille) & GU480110 \\
\hline Lecanora semipallida & & & AF070034 \\
\hline Lecanora subintricata 1 & U.S.A., Arizona & S. Pérez-Ortega 1162 (FR) & GU480111 \\
\hline Lecanora subintricata 2 & Canada, Alberta & T. Spribille 10287 (hb. Spribille) & GU480112 \\
\hline Lecanora sulphurea & & & AF070030 \\
\hline Lecanora symmicta 1 & Germany, Hessen & Ch. Printzen s.n. (FR) & GU480113 \\
\hline Lecanora symmicta 2 & & & AF070024 \\
\hline Lecanora varia 1 & & & AF070028 \\
\hline Lecanora varia 2 & & & AF070027 \\
\hline Lecanora varia 3 & & & AF070021 \\
\hline
\end{tabular}

